\title{
Solvent-dependent morphology and anisotropic microscopic dynamics of cellulose nanocrystals under electric fields
}

\author{
Kyongok Kang $\oplus^{1, *}$ and Alexey Eremin $\oplus^{2, \dagger}$ \\ ${ }^{1}$ Forschungszentrum Jülich, Institute of Biological Information Processing, \\ IBI-4:Biomacromolecular Systems and Processes, Jülich 52428, Germany \\ ${ }^{2}$ Department of Nonlinear Phenomena, Institute for Experimental Physics, \\ Otto von Güricke University Magdeburg, Magdeburg 39016, Germany
}

(Received 2 November 2020; revised 2 February 2021; accepted 3 February 2021; published 12 March 2021)

\begin{abstract}
Cellulose nanocrystals (CNCs) are interesting for the construction of biomaterials for energy delivery and packaging purposes. The corresponding processing of CNCs can be optimized through the variation of intercellulose interactions by employing different types of solvents, and thereby varying the degree of cellulose hydrogen bonding. The aim of this work is (i) to show how different types of solvents affect the self-assembled morphology of CNCs, (ii) to study the microscopic dynamics and averaged orientations on the CNCs in aqueous suspensions, including the effect of externally imposed electric fields, and (iii) to explore the nonlinear optical response of CNCs. The homogeneity of self-assembled chiral-nematic phase depends on both the polarity of the solvent and the CNC concentration. The variation of the chiral-nematic pitch length with concentration, as determined from real-space and Fourier images, is found to be strongly solvent dependent. The anisotropic microdynamics of CNCs suspension exhibits two modes, related to diffusion parallel and perpendicular to the (chiral-) nematic director. We have found also the coupling between translational and orientational motion, due to existing correlation length of twisted nematic elasticity. Preliminary second-harmonic generation experiments are performed, which reveal that relatively high field strengths are required to reorient chiral-nematic domains of CNCs.
\end{abstract}

DOI: 10.1103/PhysRevE.103.032606

\section{INTRODUCTION}

Cellulose nanocrystals (CNCs) received much scientific attention due to their versatile applications, especially in the fields concerning biodegradable and biointegrated materials, employed to bioenergy and soft electronics. The broad range of their chemical modification is connected to the variation of hydrogen-bond-induced intercellulose interactions, as well the interaction between cellulose with other types of biomolecules, for example, DNA, polysaccharides, and chitin. Since the presence of hydrogen bonds gives rise to electric conduction, very much like in DNA [1], CNCs can also be applied in ionic conductors and semiconductors [2]. A further application of cellulose is the scaffolding of tissue for regenerative biomedical substitutes [3]. It is, however, not yet clear how to tune the morphological structure of CNCbased biomaterials, and how to control the binding properties to external agents. This requires a better understanding of the inter-CNC interactions and the resulting self-assembly

\footnotetext{
*k.kang@fz-juelich.de

†alexey.eremin@ovgu.de
}

Published by the American Physical Society under the terms of the Creative Commons Attribution 4.0 International license. Further distribution of this work must maintain attribution to the author(s) and the published article's title, journal citation, and DOI. and phase behavior, depending on the solvent being used. Typically, the solubility of CNCs is crucial for the challenging tasks mentioned above. For instance, some effort was put into the fabricating of chiral-nematic cellulose nanocrystalline films, by varying the ionic strength, temperature, the concentration, and an external magnetic field [4]. Morphological control of cellulose-based systems is essential for processing and improving the performance in various kinds of applications.

CNC suspensions prepared from cellulose that is extracted from cotton and wood sources have a similar size distribution of CNCs, but differ significantly in their liquid-crystalline phase behavior. [5]. Changing the surface chemistry of the cellulose also affects the properties of corresponding CNC suspensions. The most common surface chemistry modification of cellulose in solvents involves esterification of the cellulose hydroxyl groups [6,7]. A more involved example is the enzymatic pretreatment of nanofibrilized cellulose (NFC), in combination with an external electric field in order to tune the degree of alignment and a further treatment after adding a microfluidizer, is employed in the construction of renewable materials [8]. Surface treatment is also important when CNCs are used as a reinforcing nanofiller in polymer nanocomposites, where the benefit of using CNCs is also to reduce the specific weight and to avoid degradation during melting, involved in a step in processing such nanofillers [9].

Depending on the surface chemistry, ionic strength, and $\mathrm{CNC}$ concentration, $\mathrm{CNC}$ suspensions exhibit phase 
transitions, the formation of gels and glasses, and percolation. When dispersed in an appropriate solvent, an isotropic-to(chiral-)nematic phase transition occurs at sufficiently high CNC concentration: in water, a chiral-nematic liquid crystalline ordering is observed for concentrations larger than about 3-5 wt.\% [10]. Liquid crystalline phases can also be induced by shear flow, while gels and glasses of CNCs can be formed, depending on the ionic strength and CNC concentration $[11,12]$. Aggregation and a percolation transition of CNCs are observed for surface-modified CNCs, when the number of $-\mathrm{OH}$ groups is sufficiently high, leading to an increase of the number of hydrogen bonds between the CNCs [13]. Furthermore, salt-induced structure formation in aqueous 5 wt.\% CNC suspensions is studied by means of SAXS and WAXS in Ref. [14], where it is found that the chiralnematic pitch is slightly depending on the ionic strength.

Different solvents with a high dielectric permittivity have been shown in Ref. [15] to significantly affect the kinetics of self-assembly of CNCs into helical liquid-crystalline structures and reduce the concentration dependence of the helical pitch. In this paper, we use much the same types of solvents (water, N-methylformamide, and dimethylformamide) to study the effect of these solvents on (i) the self-assembly and macroscopic morphologies and the averaged preferred orientations that are formed during phase separation, (ii) the microscopic dynamics of thermal fluctuations, (iii) the effect of externally applied electric fields, as well as (iv) the nonlinear optical response of the CNC self-assembled structures by means of second-harmonic generation. This paper is organized as follows. In Sec. II we describe the experimental methods and the systems. The results for solvent-dependent morphologies and averaged preferred orientations are given in Sec. III A, the microscopic dynamics is discussed in Sec. III B, and the observations in preliminary second-harmonic generation experiments are provided in Sec. III C. Finally, Sec. IV is a summary and conclusion.

\section{EXPERIMENTAL METHODS AND SYSTEMS}

As experimental methods, two different electric-field setups are used: (i) A home-built small-angle electric-field dynamic light scattering instrument (SAeDLS) in combination with an ITO sample cell (which will be described below) to probe the microscopic dynamics. There is no commercially vertically aligned instrument available for dynamic light scattering with small sample volumes of $300 \mu 1$, and where the weak AC electric field is applied for the small scattering angles. A photo and schematic of the setup are shown in Fig. 1(a). The vertically aligned SAeDLS setup, equipped with a He-Ne laser, probes small scattering angles $q$ in the range $q \sim 1.8-4.8 \mu \mathrm{m}^{-1}$, so that the intensity-correlation functions resolve the dynamics on length scales $2 \pi / q \sim$ 1.3-3.5 $\mu \mathrm{m}$, which is in the range of correlated length scales of CNC particles. A lens (with a focal length of $75 \mathrm{~mm}$ ) is used to detect scattered light from the center of the gap of the ITO sample cell, between the two flat electrodes. We apply a sinusoidal AC electric-field strength in the range $25-50 \mathrm{~V} / \mathrm{mm}$ (top-to-top) with a fixed frequency of $100 \mathrm{~Hz}$. A more detailed description of the SAeDLS setup can be found in Ref. [16]. (ii) The second setup is a second-harmonic generation (SHG) (a)
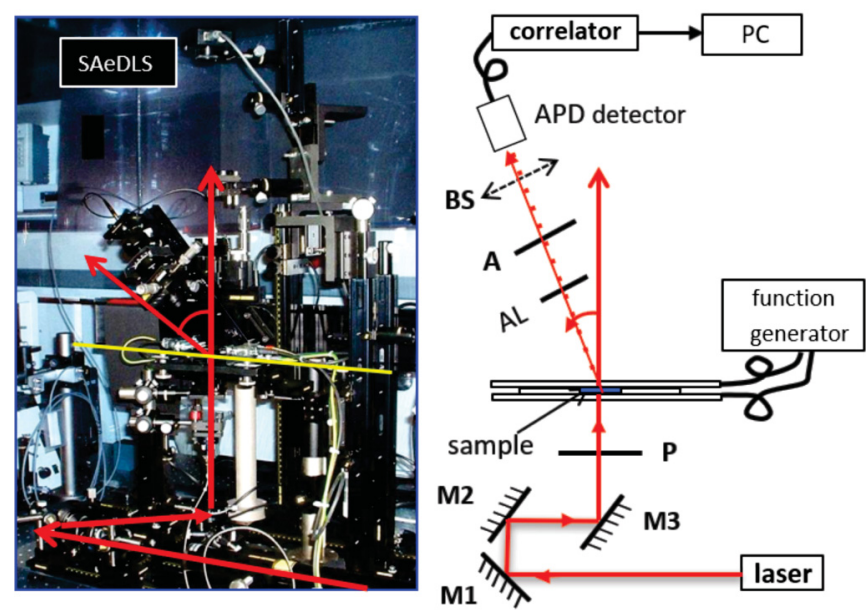

(b)

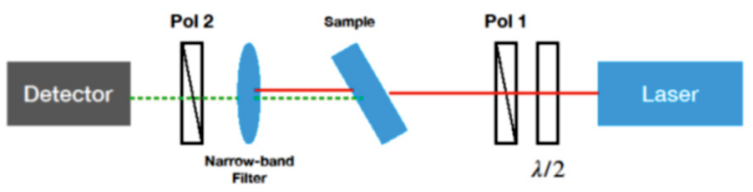

FIG. 1. (a) A photo and schematic of the small angle electricfield dynamic light scattering (SAeDLS) setup. The laser beam (from a $633 \mathrm{~nm} \mathrm{He}-\mathrm{Ne}$ laser) is indicated in red. Three mirrors (M1, M3, M3) are used to vertically align the laser beam. A polarizer (P) defines the vertical polarization of the incident beam, and the analyzer (A) is used to detect vertically polarized scattered light. An achromatic lens (AL) is used to detect scattered light only from the center in-between the two plates of the sample cell. (b) A schematic of a standard second-harmonic generation (SHG) setup. A Nd YAG $(1064 \mathrm{~nm})$ laser is used, with a $10 \mathrm{~ns}$ pulse duration and $10 \mathrm{~Hz}$ repetition rate. The optical train involves a half-lambda platelet, two polarizers, and the sample cell that is titled over $30^{\circ}$ relative to the incident laser beam. The distance between the laser and the detector is about $20 \mathrm{~cm}$, while the distances between the components are drawn on scale.

setup for the detection of nonlinear optical activity. A sketch of the setup is given in Fig. 1(b). Such a nonlinear optical response requires a much larger electric-field strength, which is achieved using in-plane switching (IPS) sample cells with a thickness of $6.5 \mu \mathrm{m}$, in combination with a Nd YAG laser. The fundamental light beam is incident at an angle of $30^{\circ}$ with respect to the normal of the cell. The SHG signal is collected in transmission direction by means of a photomultiplier tube (Hamamatsu). The effect of such large fields on the morphology is probed with an Axio Imager A.1 polarizing microscope (Carl Zeiss GmbH).

CNC wood-pulp slurries in water have been purchased from the Process Development Center at the University of Maine at a concentration of $10 \mathrm{wt} . \%$. The CNCs are hydrolyzed using sulfuric acid $\sim 64 \mathrm{wt} . \%$, which renders the CNC negatively charged, with a zeta potential in water of $-58 \mathrm{mV}$. For the solvent exchange from water to N-methylformamide (NMF) or dimethylformamide (DMF), NMF and DMF were added to a $10 \mathrm{wt} \%$ aqueous solution, followed by redispersion using high power sonication. Subsequently, the water was removed from the solution by 

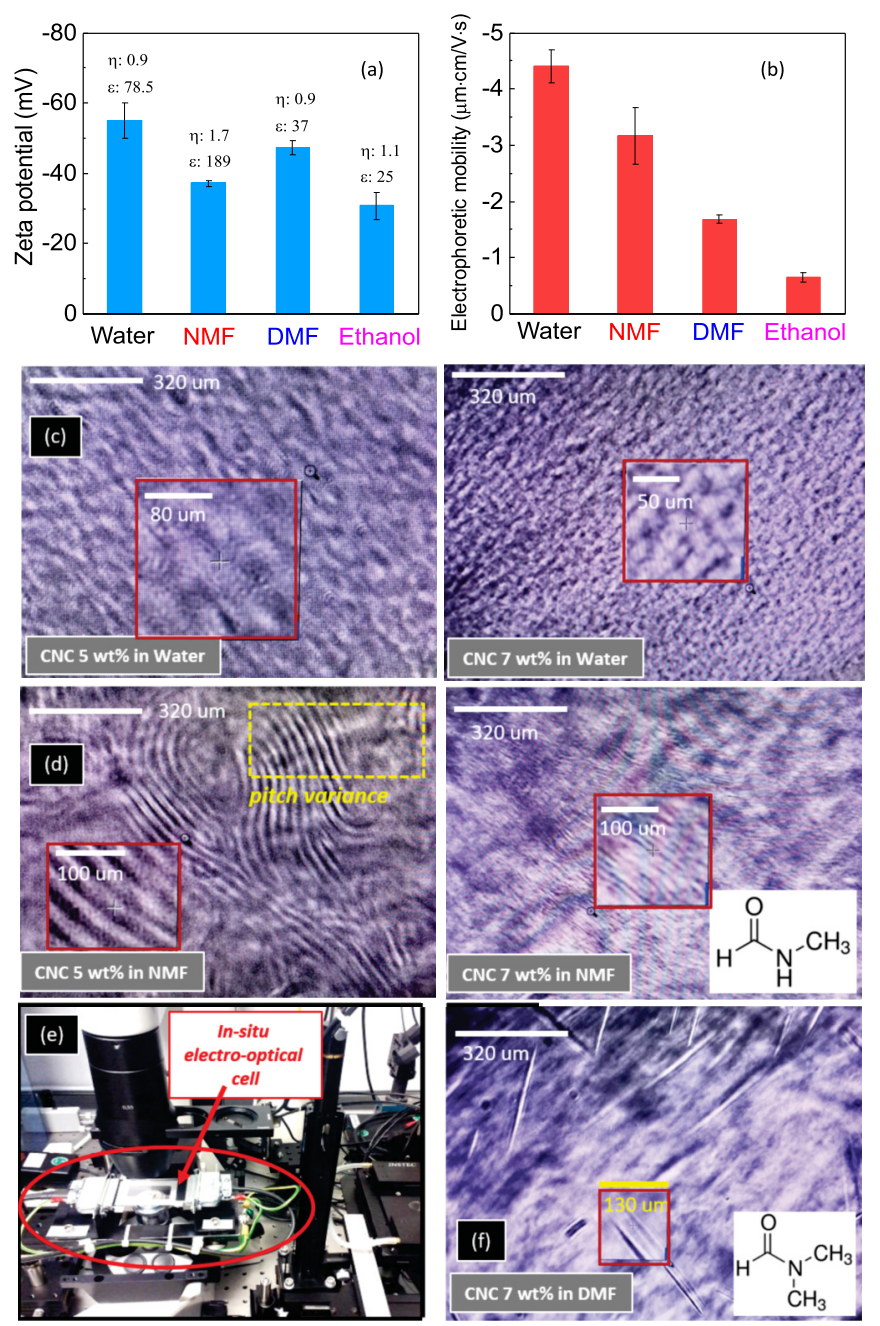

FIG. 2. (a) The zeta potential and (b) the electric mobility of CNCs dissolved in water, NMF, DMF, and ethanol, as indicated in the figures. (c), (d) Microscopy images of the morphology at 5 and 7 wt.\% CNCs in (c) water and (d) NMF. The insets show a magnified view of part of the morphology. Note that there is a large chiral-nematic pitch variance observed at the $5 \mathrm{wt} \% \mathrm{CNCs}$ in NMF in the left panel of (d). (e) A photo of the in situ ITO electric cell, mounted on an inverted microscope with which these images are taken. (f) The morphology for the most apolar solvent DMF for a concentration of $7 \mathrm{wt} . \%$. The scale bar given in (a) is $320 \mu \mathrm{m}$.

distillation at $50{ }^{\circ} \mathrm{C}$ at a reduced pressure of $50 \mathrm{mbar}$, using a rotary evaporator. The final solutions have a $\mathrm{CNC}$ concentration ranging from $9.4 \mathrm{wt} . \%$ to $10.5 \mathrm{wt} . \%$. The $\mathrm{CNC}$ suspensions remained stable in NMF and DMF for months after the solvent exchange process. The chemical structure of NMF and DMF is shown in the inset of Figs. 2(d) and 2(f), right panel, respectively. The molar mass of NMF is 59.07 $\mathrm{g} / \mathrm{mol}$ and that of DMF $73.09 \mathrm{~g} / \mathrm{mol}$. NMF and DMF are both obtained in analytical grade from Sigma-Aldrich. The zeta potentials of the CNCs and their electrophoretic mobilities are given in Figs. 2(a) and 2(b), respectively, together with the dielectric constant of these solvents. NMF is the most polar solvent, followed by water and DMF. As can be seen, the zeta potential varies between the solvents from -35 to $-58 \mathrm{mV}$.
A homemade in situ electro-optical sample cell is used, which is shown in Fig. 2(e), to probe morphologies by microscopy and measure microscopic dynamics by means of light scattering, both without and with electric fields. The cell consists of two flat indium-tin-oxide (ITO) coated glasses (from Präzisions Glas und Optik GmbH, CEC500S) with dimensions $40 \times 70 \mathrm{~mm}^{2}$, and with a thickness of $0.7 \mathrm{~mm}$. The ITO layer has a high visible light transmission ( $~ 90 \%$ at $633 \mathrm{~nm}$ ), and the ITO-coating thickness is $\sim 15 \mathrm{~nm}$. The amount of sample loaded between the two plates is $300 \mu \mathrm{l}$ within a niche of insulating PTFE (teflon) film-spacer (Armbrecht and Matthes GmbH, AR5038, and AR5038GP). The distance between the two plates is $1.0 \mathrm{~mm}$. The ITO coatings are located on the side of the sample. The measurements are done at the center of sample to ensure a homogeneous electric field. A sinusoidally varying AC electric voltage is applied to the electrodes.

\section{RESULTS AND DISCUSSION}

\section{A. Solvent-dependent morphology and the averaged preferred orientations of CNCs}

Depolarized microscopy images of morphologies, where the sample is placed between two crossed polarizers [see Fig. 2(e) for a photograph of the setup], are shown in Fig. 2. For the most polar solvent NMF, with a dielectric constant of 189, a well-ordered chiral-nematic state is observed at 5 wt.\% [see the left panel of Fig. 2(d)]. The corresponding Fourier image in Fig. 3(c) of the two-dimensional (2D) realspace morphology in the left panel of Fig. 2(d) confirms the well-ordered chiral-nematic morphology at $5 \mathrm{wt}$.\%, exhibiting bright and dark fringes as the correlation length of a chiralnematic pitch. Also, the large pitch variance is observed at the 5 wt.\% CNCs in NMF, where much smaller pitch length is also seen next to the large ones [see the yellow dashed rectangular in Fig. 2(d), left panel]. Thus, it is worthwhile to get the averaged orientations of these morphologies, via the intensity lobes in the Fourier images. Indeed, there is on average a preferred orientation of the chiral-nematic pitch in the Fourier image, such that the spatial extent of correlated $\mathrm{CNC}$ rods exist in the real-space morphology. The location of the wave vectors for alternating patterns differ by a factor of 2 (or 3), to within experimental error, due to the fact that these lobes originate from a weakly twisted elasticity in the undulating planar nematics. The halos seen in the Fourier images in Figs. 3(a)-3(d) are interpreted as being the result of remaining (background) random orientations of the chiral-nematic pitch. The halo is nonspherical since the real-space images show only the $2 \mathrm{D}$ projections of the pitch, which indicates that clearly existing spread of the pitch variances [as we have seen in Fig. 2(d)]. The pitch length is therefore obtained from the shortest distance of the halo to the center of Fourier image, as the preferred orientations of larger correlation length of chiral-nematic pitch. It is found that both for NMF and water as a solvent, the wave vectors corresponding to the location of these shortest halo distances are either equal [in Fig. 3(c) for NMF] or twice as large [in Figs. 3(a) and 3(b) for water] as the wave-vector location of the first lobe (the numbers for these locations are given in the legend to Fig. 3). This is in 

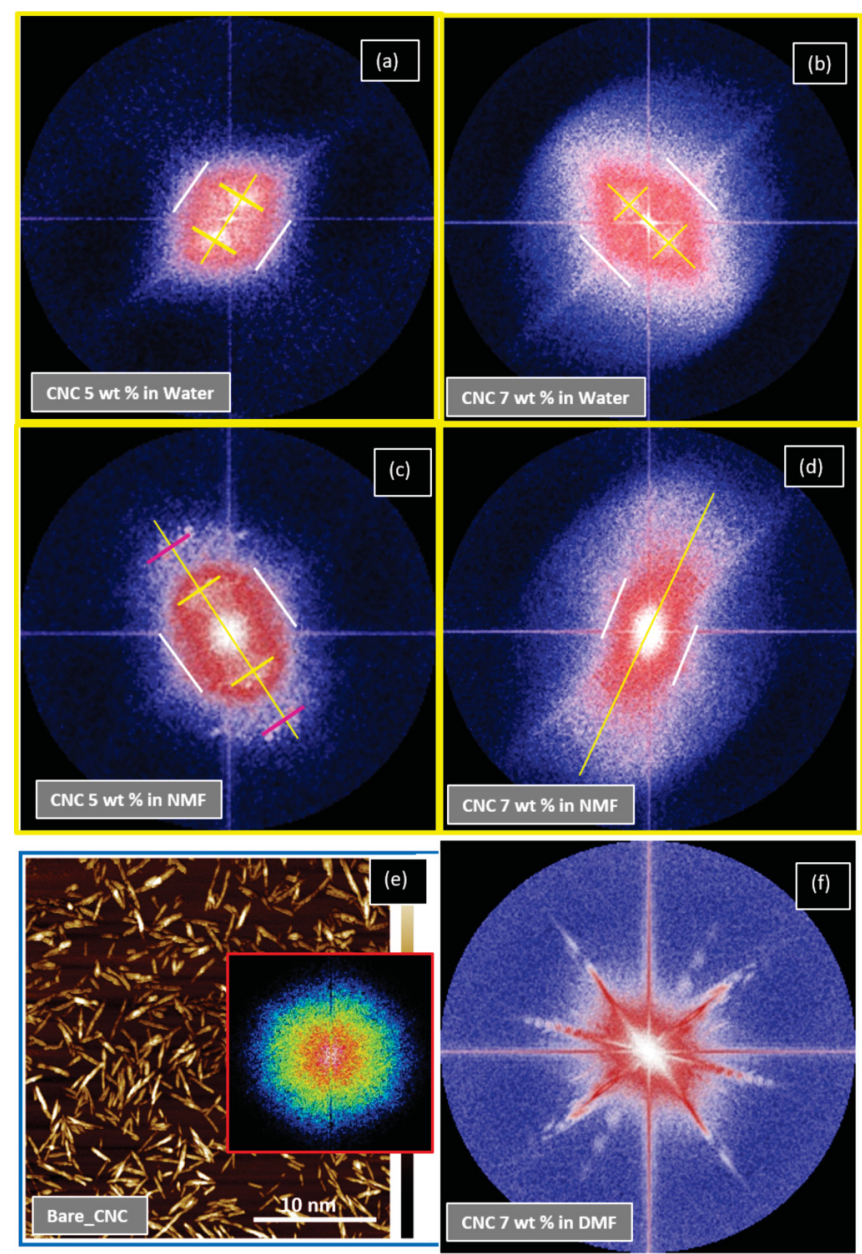

FIG. 3. Fourier transforms of the real-space 2D structural morphologies in Fig. 2 for the two concentrations 5 and 7 wt.\% in (a), (b) water, in (c), (d) NMF. (e) A TEM image of the bare CNCs. (f) The 7 wt.\% CNC dispersion in DMF. The inset is the Fourier image of the entire real-space image. The Fourier image of part of this realspace image is shown in (f), where the Fourier images of individual CNCs with random orientation can be seen. The yellow bars and the red bars in (a)-(d) relate to the location of the intensity lobes, while the white bars indicate the shortest location of the intensity halo to the center of images. The corresponding wave vectors for the yellow and red bars are (a) $0.063 \mu \mathrm{m}^{-1}$, (b) $0.076 \mu \mathrm{m}^{-1}$, (c) $0.065 \mu \mathrm{m}^{-1}$ and $0.145 \mu \mathrm{m}^{-1}$. For the white bars we have (a) $0.126 \mu \mathrm{m}^{-1}$, (b) $0.152 \mu \mathrm{m}^{-1}$, (c) $0.075 \mu \mathrm{m}^{-1}$, (d) $0.055 \mu \mathrm{m}^{-1}$. The experimental errors in these numbers are approximately $0.005 \mu \mathrm{m}^{-1}$.

accordance with the interpretation of the origin of the halos. Note that the Fourier image for the $7 \mathrm{wt} \% \%$ suspension in NMF does not show clear intensity lobes, which is due to the rather inhomogeneous morphology and the low contrast in the realspace image in Fig. 2(d), right panel. Here, the pitch of the chiral-nematic state is visibly decreased [as seen by eye from the right panel in Fig. 2(d)]. The morphology of suspension in water (as less polar than NMF) is less ordered, which can be seen from the real-space image in Fig. 2(c), leading to just an intensity lobe in the Fourier image in Fig. 3(a). Since the chiral-nematic pitch is $p=2 \pi / k$, where $k$ is the location of the wave vectors as discussed above, it is found from the averaged numerical values for the wave vectors given in the legend of Figs. 3(a) and 3(b) that the correlated pitch length is $99 \pm 5 \mu \mathrm{m}$ and $82 \pm 5 \mu \mathrm{m}$ for the 5 and $7 \mathrm{wt} . \%$ aqueous suspensions, respectively. There is thus a slight decrease of the pitch, although the macroscopic morphology is significantly changed, as can be seen from Fig. 2(c). For the NMF suspensions, there is a drastic change from $90 \pm 5 \mu \mathrm{m}$ to about $\sim 6 \mu \mathrm{m}$ [the latter is estimated from the real-space image in Fig. 2(d), right panel]. For the most apolar solvent, aggregation of the CNCs into long needles is observed, as shown in Fig. 2(f). The Fourier image is shown in Fig. 3(f), where the spikes originate from the long needlelike aggregates with different orientations, corresponding to the length of $\mathrm{CNC}$ aggregates, varied in 40-360 $\mu \mathrm{m}$.

Although the macroscopic morphology significantly changes with increasing concentration, the changes of chiralnematic pitch are found slightly for the aqueous suspensions, contrary to the NMF solutions where the larger pitch variances are observed. These findings are contrary to the result of Ref. [15], where not only the absolute value of the pitches are smaller, but there is a significant change of the pitch for aqueous suspensions on increasing the concentration, contrary to the NMF dispersion. Note, however, that the liquid-crystalline behavior of CNC suspension depends on the pretreatment and processing of cellulose source for the CNCs [5]. The chiral-nematic pitch of aqueous CNC suspension is found to be weakly dependent on the salt concentration, for even keeping the same 5 wt.\% CNCs in water [14]. Another reason is that the correlation length of chiral-nematic pitch is rather affected by the thermal fluctuation of CNCs, engaged with the longer-ranged 'twist' elasticity as less ordered on decreasing the polarity of solvent and/or increasing concentration. This may be due to the enhanced importance of hydrogen bonding, giving rise also to other aspects of short-ranged attractive interactions between the CNCs. The hydration layer surrounding the CNCs will become less structured as the dielectric constant of the solvent decreases, which allows hydrogen bonds to be formed between neighboring CNCs. This may then explain what is observed in the relatively apolar solvent DMF, with a dielectric constant of 37, where long needlelike aggregates are formed, indicative for strong attractive interactions. We have found visual observations of the morphologies, shown in Fig. 2, become more homogeneous on applying an electric field with a frequency of $100 \mathrm{~Hz}$ up to fields strengths of $50 \mathrm{~V} / \mathrm{mm}$. As will be seen in the next subsection, this slight homogenization will have a pronounced effect on the measured intensity correlation functions to probe microscopic dynamics of CNCs in water.

\section{B. Anisotropic microscopic dynamics of CNCs}

The dynamics on a length scale comparable to the correlated size of CNCs is probed by means of dynamic light scattering. We investigate CNC solutions in water for various concentrations and scattering wave vectors, in the presence of an electric field. Figure 4(a) shows the macroscopic morphology of a dispersion of 5 wt.\% in water, with a magnification in Fig. 4(b) and Fourier images in Figs. 4(c)-4(f). The Fourier image in Fig. 4(c) reveals two length scales, one related to the chiral-nematic pitch (the outer intensity lobes) and one related 


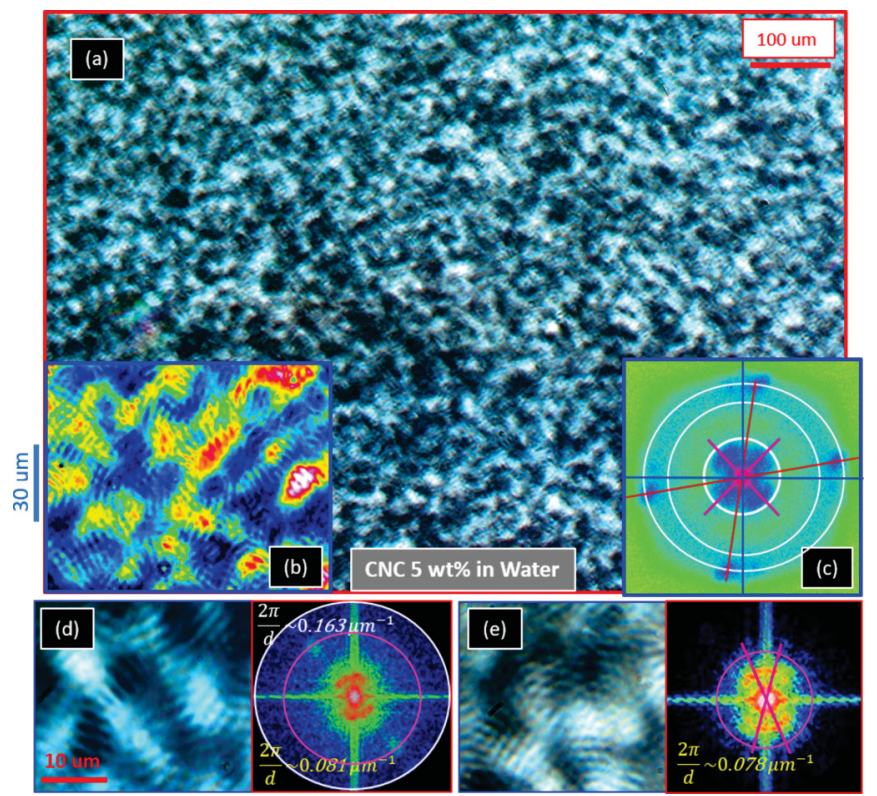

FIG. 4. (a) The macroscopic morphology of $5 \mathrm{wt} \% \mathrm{CNCs}$ in water, used in the dynamic light scattering experiments. (b) Shows a magnification of part of this macroscopic image together with the corresponding Fourier transform, which reveals the chiral-nematic domains in more detail. (c) Two different length scales are present, one corresponding to the optical pitch (the outer lobes) and the other is related to chiral-nematic domain size (the lobes near to the center). (d), (e) Different regions in real space with the corresponding Fourier images from different parts, where the averaged distribution of preferred orientations is shown in the Fourier images.

to the domain size (the lobes near the center). Time-averaged intensity correlation functions are found to have a low intercept, which are consequently quite noisy. This is attributed to the quite nonuniform morphology seen in Fig. 4: the size of the scattering volume in the dynamic light scattering experiments is of the order of $70-90 \mu \mathrm{m}$, which is of the same order of the morphological entities. The measured correlation functions are therefore dependent on the location of the scattering volume, mimicking the slow dynamics (of colloidal gels and glasses), where a single measurement probes the dynamics of entities (or microstructures) within their local environment where the laser beam is focused, which greatly varies from the true ensemble-averaged correlation function. As mentioned at the end of a previous subsection, the effect of an electric field on the morphology is to diminish to some extent nonuniformity on a macroscopic scale. The correlation functions found for samples subjected to an external electric field are evidently found to be different from the low-intercept and noisy correlation functions without the field, and independent of the position for a scattering volume.

Figures 5(a)-5(d) show normalized intensity autocorrelation functions for the four different concentrations $1,3,5$, and $7 \mathrm{wt} \%$ in water, in the presence of an electric field with frequency $100 \mathrm{~Hz}$ and a field strength of $25 \mathrm{~V} / \mathrm{mm}$, where the arrows indicate increasing wave vectors [the values of which are given in Fig. 5(a)]. The correlation functions exhibit two relaxation modes: the fast relaxation mode is related to parallel diffusion along the direction of along the $\mathrm{CNCs}$,
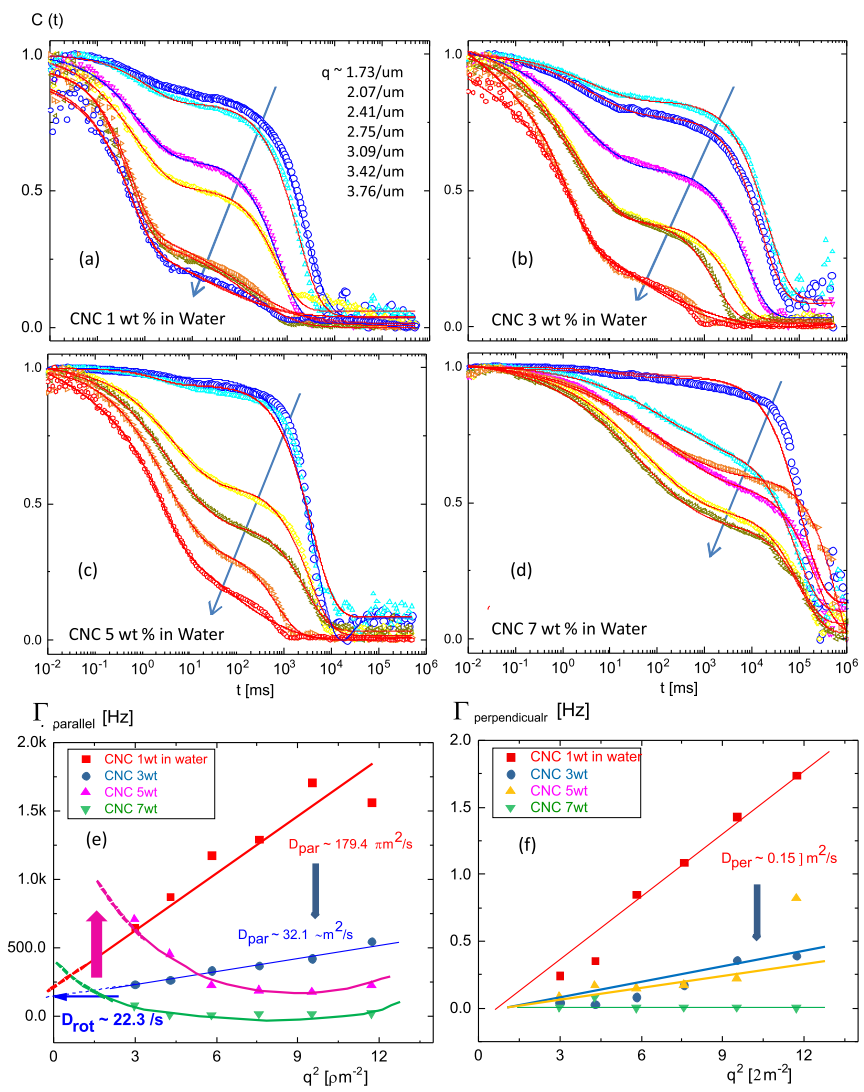

FIG. 5. Normalized intensity autocorrelations of various $\mathrm{CNC}$ concentrations in water, for CNC concentrations of (a) $1 \mathrm{wt} . \%$, (b) 3 wt. $\%$, (c) $5 \mathrm{wt} . \%$, and (d) 7 wt. $\%$. The arrow indicates increasing scattering vectors, indicated in (a). These correlation functions are measured in the presence of an external electric field with a field strength of $25 \mathrm{~V} / \mathrm{mm}$ and frequency $100 \mathrm{~Hz}$. The relaxation rates for diffusion (e) parallel and (f) perpendicular to the long axis of the CNCs.

which coincides with the local (chiral-) nematic director, and the slow mode is attributed to perpendicular diffusion. The decay rates of the two relaxation modes seen in the intensity correlation function $C(t)$ are quantified by fitting to a sum of two stretched exponential functions

$$
C(t)=B+\left[A_{f} \exp \left\{-\Gamma_{f} t\right\}^{\beta_{f}}+A_{s} \exp \left\{-\Gamma_{s} t\right\}^{\beta_{s}}\right]^{2},
$$

where $\Gamma_{f}, \Gamma_{s}$ are the relaxation rates of the fast and slow mode, for parallel and perpendicular motion, respectively, $A_{f}, A_{s}$ are the amplitudes of two modes, and $B$ is a background. The stretching parameters account for the large polydispersity, which are found to vary in the range $0.2<\beta_{f}$, $\beta_{s}<1$. The solid lines in Figs. 5(a)-5(d) are fits to the above expression. For translational diffusion, the decay rates vary as $\Gamma_{f}=D_{\|} q^{2}$ and $\Gamma_{s}=D_{\perp} q^{2}$, where $D_{\|, \perp}$ are the translational diffusion coefficients for parallel and perpendicular displacements, respectively. As can be seen from Figs. 5(e) and 5(f), the perpendicular diffusion coefficient is about a factor of a 1000 smaller than for parallel diffusion, while both decrease with increasing concentration. The diffusion coefficient for parallel motion is found to decrease from 179.4 to $32.1 \mu \mathrm{m}^{2} / \mathrm{s}$ on increasing the concentration [see Fig. 5(e)]. 

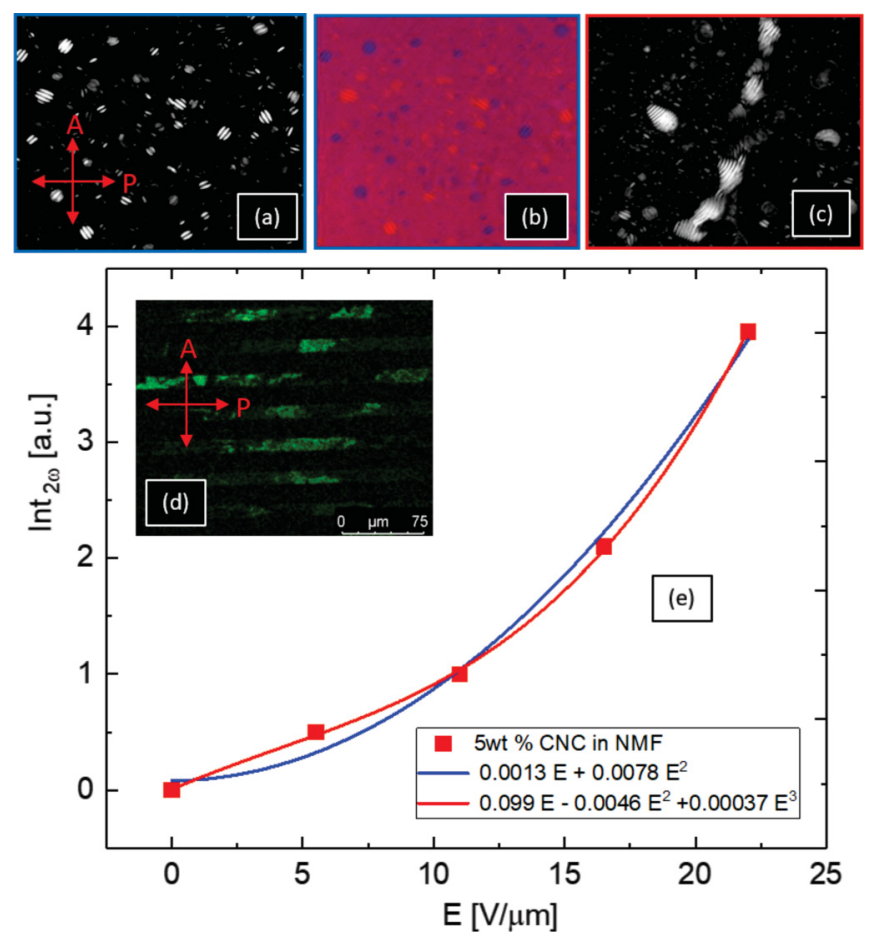

FIG. 6. (a) A depolarized light microscopy image of the chiralnematic droplets which are formed due to sonication of $6.5 \mu \mathrm{m}$ sandwich cells filled with 5 wt.\% CNC in NMF. (b) A similar image with a half-lambda platelet, and (c) a larger scale depolarized image that shows the irregular shape of the chiral-nematic droplets. (d) An image of the sample cell after a SHG experiment, revealing aggregation of $\mathrm{CNCs}$ on the electrode surface. (e) The field-strength dependence of the SHG signal. The black line is the best fit to a $\sim E^{2}$ dependence, and the red line is a best fit to a $E^{3}$ dependence.

These values are in the same range as found in Ref. [5], where it should be noted that the diffusion constants may vary with the source and preparation of CNCs. Here, we have found that the small but finite intercept at $q=0$ in Fig. 5(e) is indicative for rotational motion, with a rotational diffusion coefficient of $22 \mathrm{~s}^{-1}$, which is a few times larger than that of a cotton aggregate of CNCs $\left(3.3 \mathrm{~s}^{-1}\right)$. The diffusive behavior of relaxation modes $\sim q^{2}$ is found for all concentrations on the perpendicular diffusion, but an anomalous nondiffusive behavior is found for parallel diffusion at the two highest concentrations 5 and 7 wt.\% [see Fig. 5(e)], which confirms that the anomalous diffusion is more pronounced for smaller scattering wave vectors. Such anomalous behavior is accessed by the longer correlation length of chiral-nematic pitch length, found in the presence of an external electric field [17]. This has been now attributed to a clear coupling between rotational and translational motion of CNCs in water.

\section{Optical nonlinearity of CNCs (in NMF)}

Preliminary second-harmonic generation (SHG) experiments are performed on a sample of $5 \mathrm{wt} \%$ CNCs in NMF. Sonication of the sample within the small-gap sample cells with a gap width of $6.5 \mu \mathrm{m}$ leads to the formation of droplets, as shown in Figs. 6(a)-6(c). The size of the droplets varies from 18 to $22 \mu \mathrm{m}$, with a chiral-nematic pitch of about
$13 \mu \mathrm{m}$. Note that, although the sample is chiral nematic, at least part of the chiral-nematic structure of the droplets in Figs. 6(a) and 6(c) may be affected by the confinement, similar to what is found in the discotic liquid crystals in Ref. [18]. A weak nonlinear response is observed for field strengths up to $20 \mathrm{kV} / \mathrm{mm}$, as can be seen from Fig. 6(c). In the absence of a nonlinear optical response, in which case dipoles are induced which are proportional to the external field strength $E$, the SHG signal would be second order in the electric field strength. The data in Fig. 6(e) are fitted with a second order polynomial in $E$ (resulting in $I \sim 0.0013 E+0.0078 E^{2}$, the blue line), and a third order polynomial $\left(I \sim 0.099 E-0.0046 E^{2}+0.00037 E^{3}\right.$, the red line). The third order contribution in the $E$ dependence clearly results in a better fit, thus revealing a nonlinear optical response. The nonlinear response is due to electric field induced structural (or morphological) changes, which is evidenced by a SHG activity that does not fully relax after cessation of the electric field. Therefore, the chiral nematic domains are expected to be oriented by the relatively high electric field of a few $\mathrm{kV} / \mathrm{mm}$, in which orientation is partly "anchored" or sustained by thin ITO sample-cell confinement. More quantitative investigations are then appropriate to assess the role played by the solvent and the effects of confinement and wall$\mathrm{CNC}$ interactions in the nonlinear optical response of $\mathrm{CNC}$ suspensions.

\section{SUMMARY AND CONCLUSION}

We investigated the solvent-dependent macroscopic structures for three different solvents, N-methylfomamide (NMF), water, and dimethylformamide (DMF), with decreasing polarity: the relative dielectric constants are 189, 80, and 37, respectively. For the two most polar solvents, a chiral-nematic phase self-assembles, with different macroscopic morphologies. The larger degree of disorder for the less polar solvent is due to the diminished dielectric screening of the surface charges of the CNCs. Such a less effective dielectric screening is due to the smaller degree of alignment for solvent molecules at the surface of CNCs, which allows the formation of hydrogen bonds between CNCs. The degree of nonuniformity in the morphology varies with increasing CNC concentration, which can be understood in terms of an increased tendency to form hydrogen bonds at such higher packing fractions, as well the correlation length and their preferred averaged orientation of chiral-nematic state for interacting CNCs. That hydrogen bonding plays an important role and it explains the formation of elongated aggregates for the low-dielectric solvent DMF. The rodlike CNC aggregates are found to have a very broad length distribution of 40-360 $\mu \mathrm{m}$.

The anisotropic microscopic dynamics of CNCs in water has been probed with small angle electric-field dynamic light scattering. Without an electric field, the intensity autocorrelation functions have a very low intercept, and are consequently quite noisy, depending on the location of a scattering volume. Since the morphology of the samples varies on the correlation length scale similar to the linear dimensions of the scattering volume, each measurement probes the microscopic dynamics of CNCs in the location of a static scattering volume. In the presence of an electric field, however, these features 
are essentially absent due to a field-induced homogenization of the averaged thermal fluctuations of CNCs. The intensity correlation functions exhibit two translational diffusive decay modes, a fast mode due to diffusion parallel to the CNCs long axis, and a slow mode for perpendicular motion. Both diffusion coefficients for parallel and perpendicular motion decrease by orders of magnitude on increasing the concentration from $1 \mathrm{wt} . \%$ to $7 \mathrm{wt} . \%$. For very high concentrations, an anomalous wave vector dependence is found for the fast mode, which is the coupling of rotational and translational motion, attributed to the very small twist elasticity in the correlation length of chiral nematics. According to the secondharmonic generation experiment, quite high electric fields (of the order $\mathrm{kV} / \mathrm{mm}$ ) are required to reorient CNCs. Such a field-induced orientation leads to nonlinear optical response, which persists after switching off the field. It seems that still systematic experiments are useful in the possible application of CNCs to create nonlinear optical materials; for instance, the reversible electric-field-induced alignment of CNCs can be checked by the field effect on the "tuning" of polarity of CNCs in the planar-nematic state.

Thus, the above mentioned findings may be a starting point for further studies on CNC suspensions in various solvents and in electric fields, including the manufacturing of biodegradable materials. Systematic studies are then required where the electric-field strength is varied from several $\mathrm{V} / \mathrm{mm}$ up to several $\mathrm{kV} / \mathrm{mm}$, as an intermediate to the field strengths we have addressed in this paper. At the high field strengths, we then expect an appreciable effect on the orientation and morphology of chiral-nematic CNCs, possibly leading to correlated aggregation. The small length scale dynamics of CNCs proceeds on a millisecond to second timescale, which are comparable to timescales related to the switching time between bistable (or tristable) states on application or cessation of an electric field, as relevant for nonlinear optics integrated CNCs. We finally hope this work will stimulate additional work on the characteristics of chiral-nematic morphology depending on the polarity of the solvent, the effects of electric fields, and the microscopic dynamics, which might contribute to a rational approach towards the processing of ecologically friendly CNC-based materials.

\section{ACKNOWLEDGMENTS}

The authors thank Dr. I. S. Chae for providing the samples and Prof. J. K. G. Dhont for his critical reading of the manuscript and further discussions on the roles of hydrogen bonding in CNCs at different solvents.
[1] C. Dekker and M. A. Ratner, Phys. World 14, 29 (2001).

[2] E. D. Glowacki, M. Irimia-Vladu, S. Bauer, and N. S. Sariciftci, J. Mater. Chem. B 1, 3742 (2013).

[3] F. Afghah, C. Dikyol, M. Altunbek, and B. Koc, Appl. Sci. 9, 3540 (2019).

[4] J. Pan, W. Hamad, and S. K. Strauss, Macromolecues 43, 3851 (2010).

[5] C. Schuetz, J. van Rie, S. Eyley, and A. Gencer, Sustainable Chem. Eng. 6, 8317 (2018).

[6] P. M. Kosaka, A. Jr. J. Amin, R. S. N. Saito, and D. F. S. Petri, ACS. Sym. Ser. 1019, 223 (2009).

[7] K. J. Edgar, C. M. Buchanam, J. S. Debnenham, P. A. Rundquist, B. D. Seiler, M. C. Shelton, and D. Tindall, Prog. Polym. Sci. 26, 1605 (2001).

[8] A. Kadimi, K. Benhamou, Z. Ounaises, A. Magnin, A. Dufresne, H. Kaddami, and M. Raihane, Appl. Mater. Interface 6, 9418 (2014).

[9] M. Mariano, F. Pilate, F. B. de Oliveira, F. Khelifa, P. Dubois, J.-M. Raquez, and A. Dufresne, ACS Omega 2, 2678 (2017).
[10] J-F. Revel, H. Bradford, J. Giasson, R. H. Marchessault, and D. G. Gray, Int. J. Biol. Macromol. 14, 170 (1992).

[11] Y. Xu, A. D. Atrens, and J. R. Stokes, J. Coll. Int. Sci. 496, 130 (2017).

[12] Y. Xu, A. D. Atrends, and J. R. Stokes, Soft Matter 14, 1953 (2018).

[13] L. S. Blachechen, J. P. de Mesquita, E. L. de Paula, F. V. Pereira, and D. F. S. Petri, Cellulose 20, 1329 (2013).

[14] P. Bertsch, A. Sánchez-Ferrer, M. Bagnani, S. Isabettini, J. Kohlbrecher, R. Mezzenga, and P. Fischer, Langmuir 35, 4117 (2019).

[15] J. R. Bruckner, A. Kuhnhold, C. Honorato-Rios, T. Schilling, and J. P. F. Lagerwall, Langmuir 32, 9854 (2016).

[16] K. Kang, Rev. Sci. Instrum. 82, 053903 (2011).

[17] K. Kang, P. Bertsch, and P. Fischer, Phys. Rev. E. 100, 052606 (2019).

[18] A. Yildirim, K. Sentker, G. J. Smales, B. R. Pauw, P. Huber, and A. Schönhals, Nanoscale Adv. 1, 1104 (2019). 